\title{
Detection of Tumours in Digital Mammograms Using Wavelet Based Adaptive Windowing Method
}

\author{
G.Bharatha Sreeja \\ PG Communication Systems, Cape Institute of Technology, Levengipuram, India \\ Email: bharathasreeja@yahoo.com \\ Dr. P. Rathika \\ Professor, ECE Dept., Cape Institute of Technology, Levengipuram, India \\ Email: rathikasakthikumar@yahoo.co.in \\ Dr. D. Devaraj \\ DEAN, R\&D, Kalasalingam University, Krishnankoil, India \\ Email: deva230@yahoo.com
}

\begin{abstract}
Mammography is the most effective procedure for the early detection of breast diseases. Mammogram analysis refers the processing of mammograms with the goal of finding abnormality presented in the mammogram. In this paper, the tumour can be detected by using wavelet based adaptive windowing technique. Coarse segmentation is the first step which can be done by using wavelet based histogram thresholding where, the thereshold value is chosen by performing 1-D wavelet based analysis of PDFs of wavelet transformed images at different channels. Fine segmentation can be done by partitioning the image into fixed number of large and small windows. By calculating the mean, maximum and minimum pixel values for the windows a threshold value has been obtained. Depending upon the threshold values the suspicious areas have been segmented. Intensity adjustment is applied as a preprocessing step to improve the quality of an image before applying the proposed technique. The algorithm is validated with mammograms in Mammographic Image Analysis Society Mini Mammographic database which shows that the proposed technique is capable of detecting lesions of very different sizes.
\end{abstract}

Index Terms - wavelet based Thresholding, breast cancer, mammography, window based Thresholding, segmentation.

\section{INTRODUCTION}

Currently, breast cancer is a leading cause of death among women and second major cause of death after lung cancer [1]-[5]. Breast cancer is the second most common cancer in Indian women. The incidence is more in urban than rural women. It is more prevalent in the higher socio-economic groups. The average incidence rate varies from 22-28 per 1,00,000 women per year in urban settings to 6 per 100,000 women per year in rural areas. Due to rapid urbanization and westernization of lifestyles, there is a rising incidence of breast cancer in India. According to The International Agency for Research on Cancer, which is part of the World Health Organization, there were approximately 79,000 women per year affected by breast cancer in India. It is thought that it takes about 10 years for a tumour to become $1 \mathrm{~cm}$ in size starting from a single cell. Earlier diagnoses of breast cancer are of great importance in modern medicine.

At present, mammography is the method of choice for early breast cancer detection [6]-[8]. Although automatic analysis of mammograms cannot fully replace radiologists, an accurate computer-aided analysis method can help radiologists to make more reliable and efficient decisions [9]. Tumors and other abnormalities present in the mammograms are of basic interests that need to be segmented and extracted in mammograms [10]-[11]. Some of the grayscale based segmentation methods are quite effective to extract the exact edges of homogeneous grayscale regions. They are often not so effective to extract the desired affected areas in mammograms with complex structure because of the complex distribution of the grayscale. However, the appearances of breast cancers are very subtle and unstable in their early stages. Therefore, doctors and radiologists can miss the abnormality easily if they only diagnose by experience. The computer aided detection technology can help doctors and radiologists in getting a more reliable and effective diagnosis. There are numerous tumour detection techniques have 
been analyzed [12]-[13].A region-growing technique has been discussed by Umesh Adiga et al [14] which is constrained by shape and size similarities of cell nuclei. In high throughput tissue image analysis, this method requires an automatic initial seed selection and becomes slow due to the requirement of continuous updating of similarity measures. The same issues limit the semi-automation method proposed by $\mathrm{Wu}$ and Barba [15]. When morphological filters are used, the shape of the segmented object tends to change in accordance with the shape of the structuring elements used for filtering [16]-[18]. Wu et al. proposed a parametric model-fitting algorithm for cell segmentation [19]. In this method, they assume objects are convex and hence a shape model can be used for segmentation. The composition of the algorithm is also very complicated when overlapped structure are present in the image.

Wavelet transform-based methods offer a natural framework for providing multiscale image representations that can be separately analyzed [20][25].Through a multiscale decomposition [26]-[28], most of the gross intensity distribution can be isolated in a large scale image, while the information about details and singularities, such as edges and textures, can be isolated in mid- to small scales. Here 1-D wavelet-based analysis is performed to find the PDF and adaptively selected proper thresholds for segmentation by searching for the local minima of the 1-D wavelet transformed PDF [29]. This method is simple, fast, and effective for segmenting tumors in mammograms. However, the method is not very effective when the target and the background regions demonstrate little difference in gray-level values. According to the neighboring windows around the pixel a threshold is computed for each pixel of the image. It did not consider the case where a mass contains the small window, the center region of a suspicious lesion is not detected, and it gives an empty area in the segmentation result. In other words, the algorithm can obtain good detection results on one type of lesions, but it may generate unreasonable detection results on other types of lesions.

An approach is used to segment the suspicious mass regions by a local adaptive thresholding technique [30]-[32] after the mammograms are enhanced with a linear transformation filter. For each pixel of the image, a threshold is computed according to the neighboring windows around the pixel. Next, a decision is made to classify the pixel whether it belongs to a suspicious lesion or a normal region by the threshold. From the experimental results, we can see that this algorithm works well in mammographic mass detection. At the same time, experiments show that the algorithm has a shortage: It did not consider the case where a mass contains the small window, the center region of a suspicious lesion is not detected, and it gives an empty area in the segmentation result.

Global thresholding is one of the common techniques [33] for image segmentation. It is based on the global information, such as histogram. The fact that masses usually have greater intensity than the surrounding tissue can be used for finding global threshold value. On the histogram, the regions with an abnormality impose extra peaks while a healthy region has only a single peak. After finding a threshold value the regions with abnormalities can be segmented [34][35]. Global thresholding is not a very good method to identify ROI (Region of Interest) because masses are often superimposed on the tissue of the same intensity level.

In this paper, wavelet based adaptive windowing method is proposed with multiple thresholds for separating the suspicious areas. Thresholds are chosen adaptively for segmenting tumours. Regions having gray levels below the threshold are assigned as background and regions having gray levels above the threshold are assigned as suspicious regions. In this work, the mammograms obtained from the Mammographic Image Analysis Society (MIAS) Mini Mammographic database are used to test the proposed algorithm. The experimental results show that the proposed algorithm works effectively for mammographic image segmentation.

In this paper, the work is structures as follows. Section 2, describes the proposed method for segmenting breast cancer. Section 3 discussed about the results. Finally, Section 4 discusses the simulation results.

\section{PROPOSED METHOD}

In this paper, the tumour can be detected by using wavelet based adaptive windowing technique. Flow chart for the proposed technique is given in Fig.1.The following are the major stages of computation used to segment the tumours in digital mammograms:

\section{A.PREPROCESSING}

The aim of pre processing is to improve the image data by suppressing the undesired distortions or enhances some image features relevant for further processing and analysis task. In this paper, linear contrast stretching is used as a preprocessing step. This is the simplest contrast stretch algorithm. The gray values in the original image and the modified image follow a linear relation in this algorithm. A value in the low range of the original histogram is assigned to extremely black and a value at the high end is assigned to extremely white. 


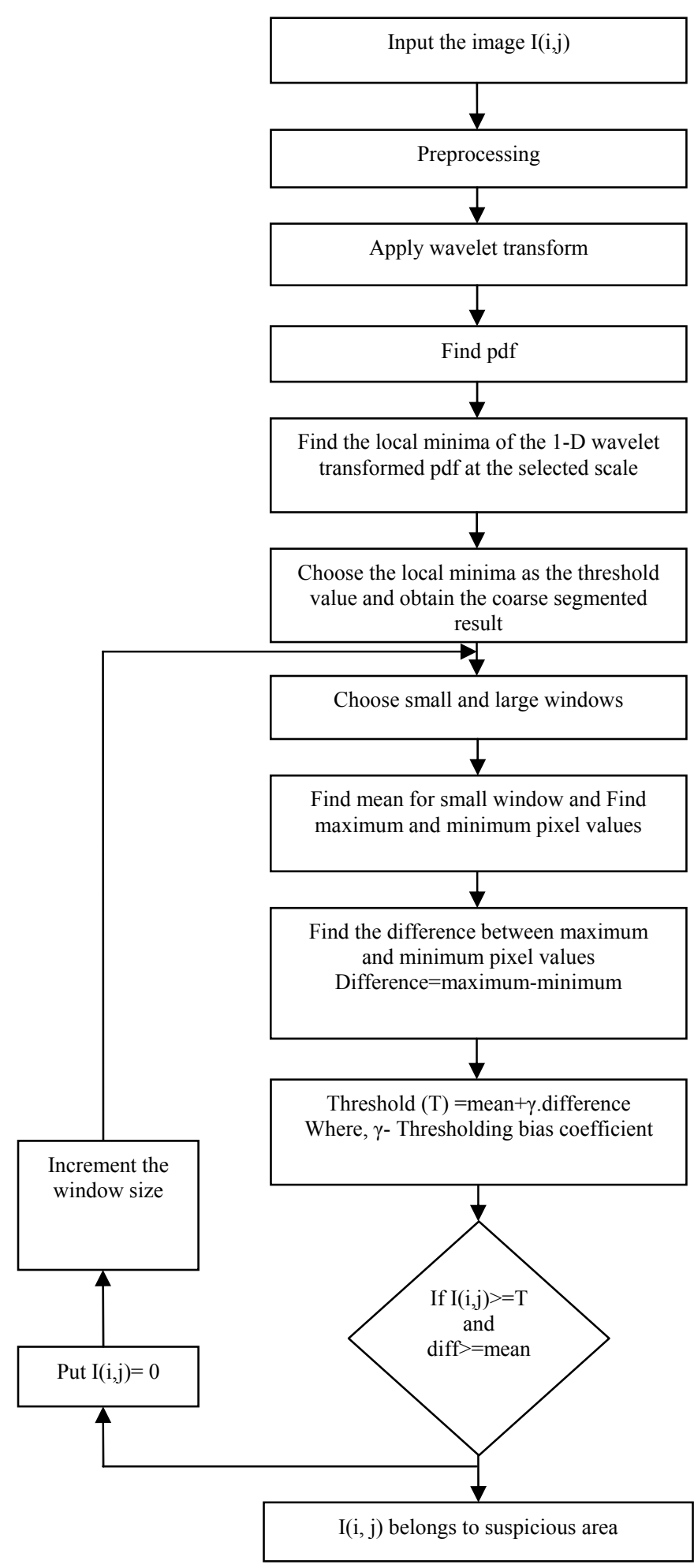

Figure 1. Flow chart of the wavelet based adaptive windowing method of segmentation

The remaining pixel values are distributed linearly between these extremes. The features that were obscure on the original image will be clear in the contrast stretched image. The pixel values are changed to new values after applying preprocessing.

\section{B.COARSE SEGMENTATION}

Segmentation subdivides an image into its constituent regions or objects that have similar features according to a set of predefined criteria. In this paper, the rough segmentation is done by using wavelet based histogram thresholding where, the thereshold value is chosen by performing 1-D wavelet based analysis of PDFs of wavelet transformed images at different channels.

\section{(i)Review of Wavelet transform}

The Discrete Wavelet Transform (DWT) of image signals produces a non-redundant image representation, which provides better spatial and spectral localization of image formation. The DWT can be interpreted as signal decomposition in a set of independent, spatially oriented frequency channels. The signal is passed through two complementary filters and emerges as two signals, approximation and details. This is called decomposition.

Fig.2. shows the bank of filters iterated for the 2DDWT standard. The components can be assembled back into the original signal without loss of information. This process is called reconstruction or synthesis. The mathematical manipulation, which implies analysis and synthesis, is called discrete wavelet transform and inverse discrete wavelet transform. An image can be decomposed into a sequence of different spatial resolution images using DWT. In case of a 2D image, an $\mathrm{N}$ level decomposition can be performed resulting in $3 \mathrm{~N}+1$ different frequency bands namely, LL, LH, HL and HH as shown in Fig.3. The next level of wavelet transform is applied to the low frequency sub band image LL only. The Gaussian noise will nearly be averaged out in low frequency wavelet coefficients. Therefore, only the wavelet coefficients in the high frequency levels need to be thresholded.

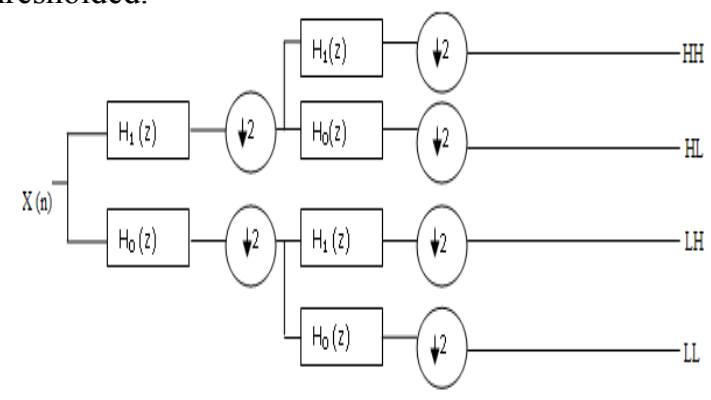

Figure 2. Bank of filters iterated for the DWT standard

In this paper, the concepts of Daubechies wavelet transform are discussed. The Daubechies wavelets are a family of orthogonal wavelets defining a discrete wavelet transform and characterized by a maximal number of vanishing moments for some given support. With each wavelet type of this class, there is a scaling function which generates an orthogonal multi resolution analysis. Daubechies wavelets are widely used in solving a broad range of problems, e.g. selfsimilarity properties of a signal or fractal problems, signal discontinuities, etc. 


\begin{tabular}{|c|c|c|c|}
\hline $\mathbf{L L}^{3}$ & $\mathbf{L H}^{3}$ & & \\
\hline $\mathbf{H L}^{3}$ & $\mathbf{H H}^{3}$ & $\mathbf{L H}^{2}$ & \\
\hline & & $\mathbf{H H}^{2}$ & \\
\hline \multicolumn{3}{|c|}{$\mathbf{H L}^{1}$} & $\mathbf{H H}^{1}$ \\
\hline
\end{tabular}

1, 2, 3 --- Decomposition levels

$\mathrm{H}$--- High Frequency Bands

L --- Low Frequency Bands

Figure 3. 2D-DWT with 3-level decomposition

The decomposition of the image into different resolution levels which are sensitive to different frequency bands. By choosing an appropriate wavelet with a right resolution level, tumours can be detected effectively in digital mammogram. Experimental results show that the Daubechies wavelet achieves the best detecting result [34].

(ii)Wavelet based Thresholding

After preprocessing, the daubechies wavelet transform is applied to a preprocessed image. Proper scaling channel is chosen using prior information of appropriate size of the target. After applying wavelet transform, find the histogram. Then perform 5 scale 1D db-6 wavelet transform. Calculate the local minima of the 1-D wavelet transformed pdf at the selected scale. The threshold value is selected by using that local minima value. Then segmentation is done by using threshold value to obtain the coarse segmented areas.

\section{C.FINE SEGMENTATION}

\section{(i)Window Selection}

The first step in fine segmentation is the selection of small and large windows. Here the whole image $\mathrm{R}$ is partitioned into a finite set of large regions or large windows R1, R2,....., Rm.

$$
\mathrm{R}=\bigcup_{i=1}^{m} R i \text { Where, } \mathrm{i}=1,2, \ldots, \mathrm{m}
$$

Then small window is chosen within the large window $\mathrm{Ri}$ and then find threshold for each window. Theoretically, the smaller the window size is, the better the result will be. However, when the window size becomes too small, it can produce the problem of homogenous windows, i.e., windows contain only background or object pixels. As a consequence, black areas called ghost objects will occur after thresholding. Therefore, there is a need to develop a correct window size in order to obtain optimal result. Final segmentation depends on the proper selection of initial window size.

\section{(ii)Window based Thresholding}

Fig. 1 shows the flowchart of the proposed method for tumour detection. For each pixel in coarse segmented result $I(i, j)$, a decision is made to classify it into a potential suspicious lesion pixel or a normal pixel by the following rule.

If $\mathrm{I}(\mathrm{i}, \mathrm{j}) \geq \mathrm{TH}(\mathrm{i}, \mathrm{j})$ and Difference $\geq$ Mean, then $\mathrm{I}(\mathrm{i}, \mathrm{j})$ belongs to the suspicious area; else, $I(i, j)$ belongs to the normal area. In this rule, $\mathrm{TH}(\mathrm{i}, \mathrm{j})$ is an adaptive threshold value calculated by equation(4).Here, $\gamma$ is a thresholding bias coefficient. Its value ranges from zero to one. Mean is an average of pixel intensity in a small window around the pixel $I(i, j) ; \max (i, j)$ and $\min (i, j)$ are the maximum and minimum intensity values in the large window. Each step is described further below.

1).Consider the coarse segmented image $I(i, j)$ and choose the window sizes of (w1 x w1) \& (w2 x w2) for large window and small window respectively.

2).Determine the mean for small window using the equation

$$
\text { Mean }=\frac{1}{N} \sum_{i, j=1}^{m} S(i, j)
$$

where, $\mathrm{N}$ is the total number of pixels in small window, $\mathrm{S}(\mathrm{i}, \mathrm{j})$ is the pixel value within the small window.

3).Compute the maximum and minimum pixel values within the large window.

$$
\begin{aligned}
& \operatorname{Maximum}=\max (L(i, j)) \\
& \operatorname{Minimum}=\min (L(i, j))
\end{aligned}
$$

where, $L(i, j)$ is the pixel value within large window.

4).Find the difference between maximum and minimum pixel values.

$$
\text { Difference }=\max (L(i, j))-\min (L(i, j))
$$

5).Calculate the threshold value by using the following equation

$$
\mathrm{TH}(\mathrm{i}, \mathrm{j})=\text { Mean }+\gamma \cdot \text { difference }
$$

with difference $=\max (i, j)-\min (i, j), \gamma$ is a thresholding bias coefficient

6).If $I(i, j) \geq T H(i, j)$ and Difference $\geq$ Mean, then $I(i$, j) belongs to the suspicious area; else, $I(i, j)$ belongs to the normal area otherwise, shift the window by one step and goto step (2)

7).Repeat steps (2)-(6) till the whole image is exhausted. 


\section{III. RESULTS AND DISCUSSION}

The proposed method is tested by using the miniMIAS database of mammograms. All images are digitized at the resolution of $1024 \times 1024$ pixels and 8 bit accuracy (gray level). The testing images include 209 normal images, 23 images of CIRC (Circumscribed masses), 19 images of SPIC(Speculated masses), 19 original images of MISC(ill-defined masses), 23 images of CALC(Calcification). The proposed algorithm was implemented in a MATLAB environment.

The original image is shown as Fig.4(a). The preprocessing is done by linear contrast stretching which is shown in Fig.4(b). Daubechies 6-point wavelet is selected to process the image.

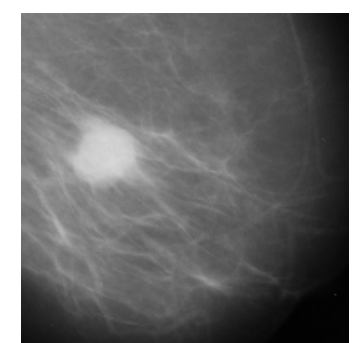

(a) Original image

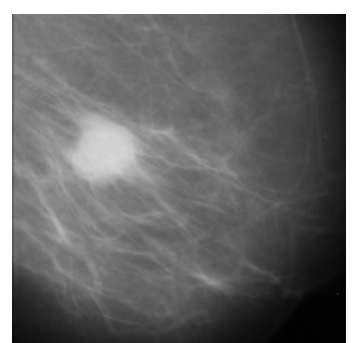

(b)Preprocessed image
Figure 4. Original image and preprocessed image

Fig.5 (a)-(d) shows the wavelet transforms of the input image from scales 1 to 4 . Then histogram is taken for transformed images. Fig.6 (a)-(d) indicates the histograms (PDFs) of these four transformed images respectively. The image in scale 2 is used for segmentation since it can effectively detect the tumours present in the digital mammograms. Next, 5-scale wavelet transforms for the histogram of the image in scale 2 is taken.

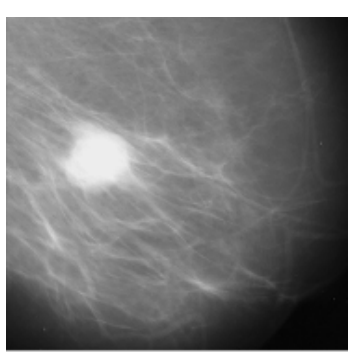

(a)

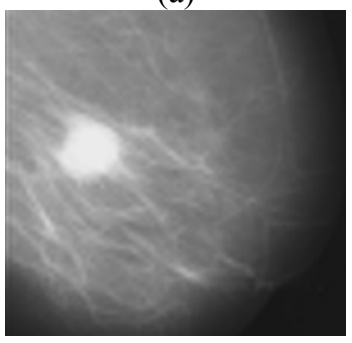

(c)

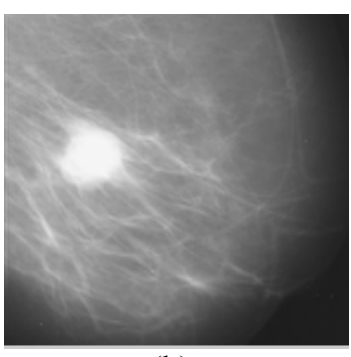

(b)

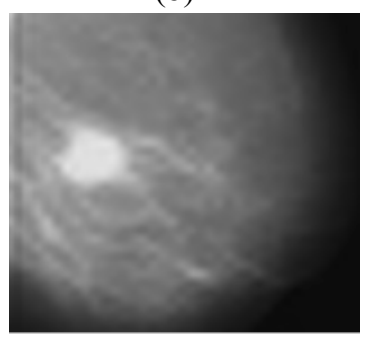

(d)
Figure 5. Images of four scaling channels of DWT

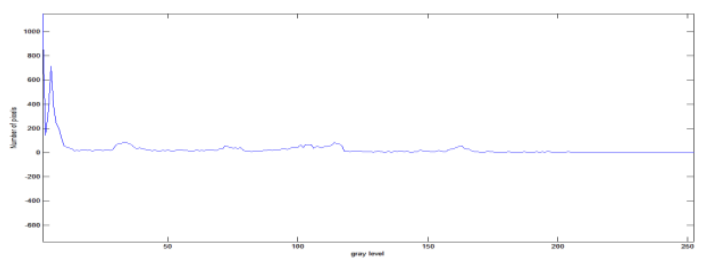

(a)Histogram of first scaling channel

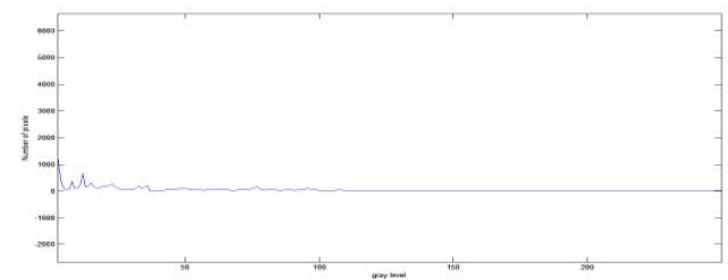

(b)Histogram of second scaling channel

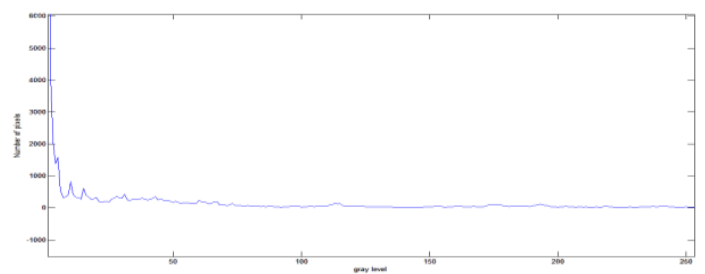

c) Histogram of third scaling channel

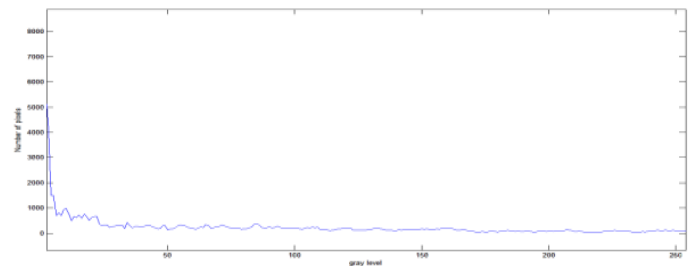

(d) Histogram of fourth scaling channel

Figure 6. Histograms (PDFs) of the four transformed images

By taking the local minima of the curve at adaptively selected scale, four local minima are obtained. Using the largest local minimum as the adaptive threshold, the coarse segmented areas are obtained. In order to show the superiority of the proposed method it is compared with global thresholding and window based adaptive thresholding method. Global threshold methods suffer from drawback as threshold value is fixed manually. Some of them can be segmented accurately and part the tumour cannot be detected correctly.

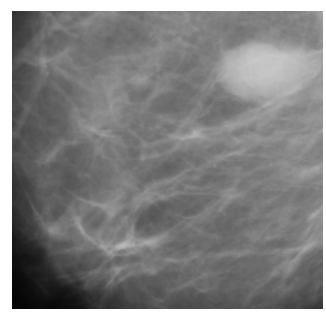

(a) Original image

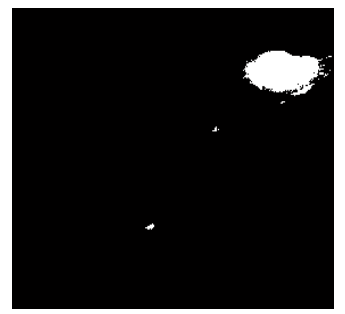

(b) Segmented result Image mdb025 


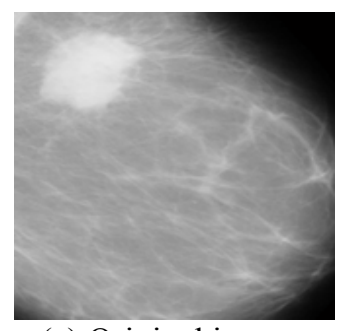

(a) Original image Image mdb184

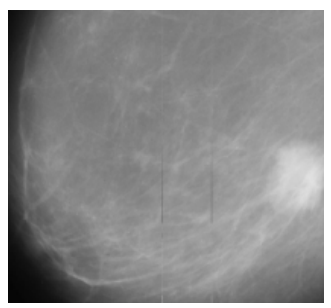

a) Original image

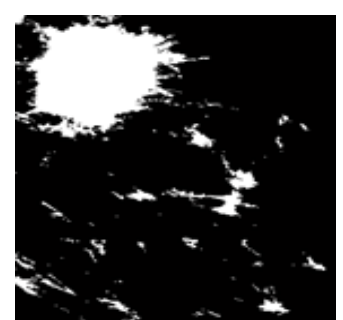

(b) Segmented result

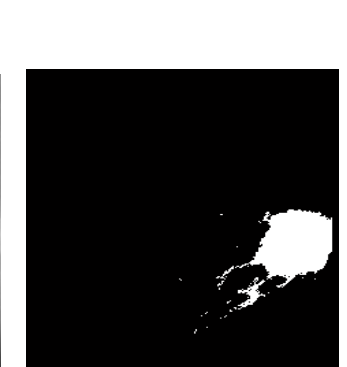

b) Segmented result Image mdb271

Figure 7. Example of segmentation results by the Global thresholding

For example Fig.7 (a) shows the original mammogram images. Then Fig.7 (b) shows the bad segmentation results because it cannot detect the entire tumour area. Global thresholding is not a very good method to identify ROI (Region of Interest) because masses are often superimposed on the tissue of the same intensity level. Simulation results show that the proposed algorithms yield significantly superior image quality when it is compared to the Global thresholding method and window based adaptive thresholding method.

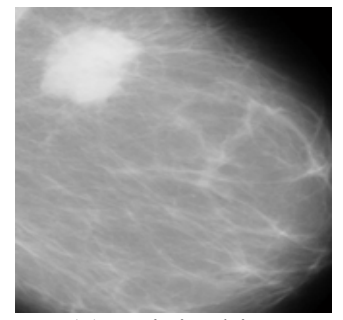

(a) Original image

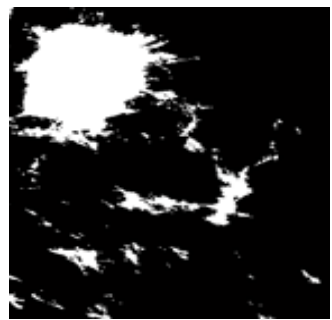

Image mdb184

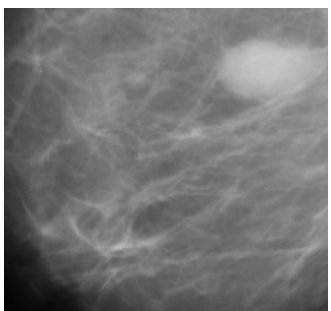

(a) Original image

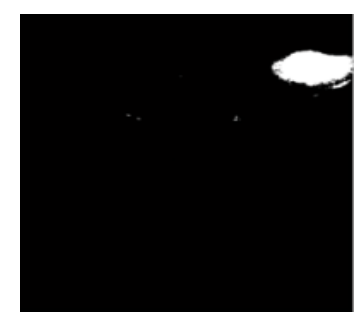

(b) Segmented result Image mdb025

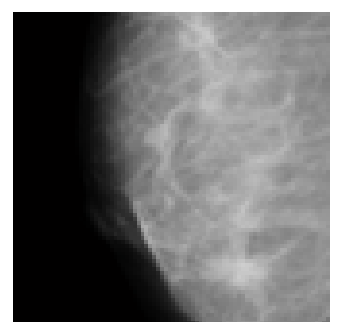

(a) Original image

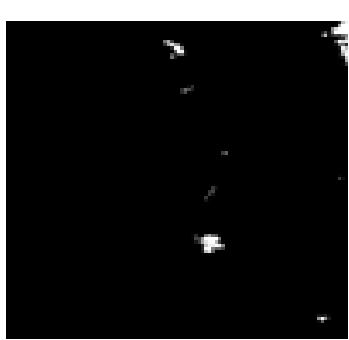

(b) Segmented result mdb181
Figure 8. Example of segmentation results by the window based adaptive thresholding segmentation

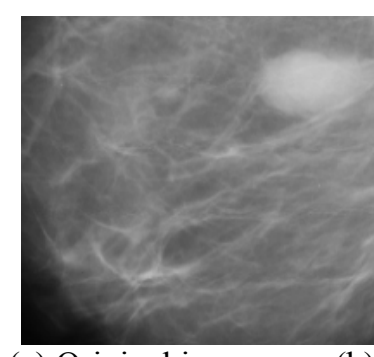

(a) Original image

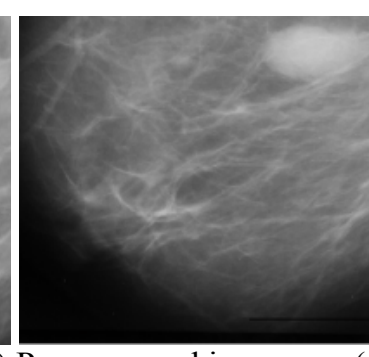

(b) Preprocessed image

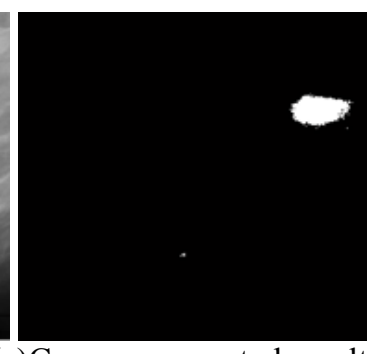

(c)Coarse segmented result (d)Fine segmented result Image mdb025
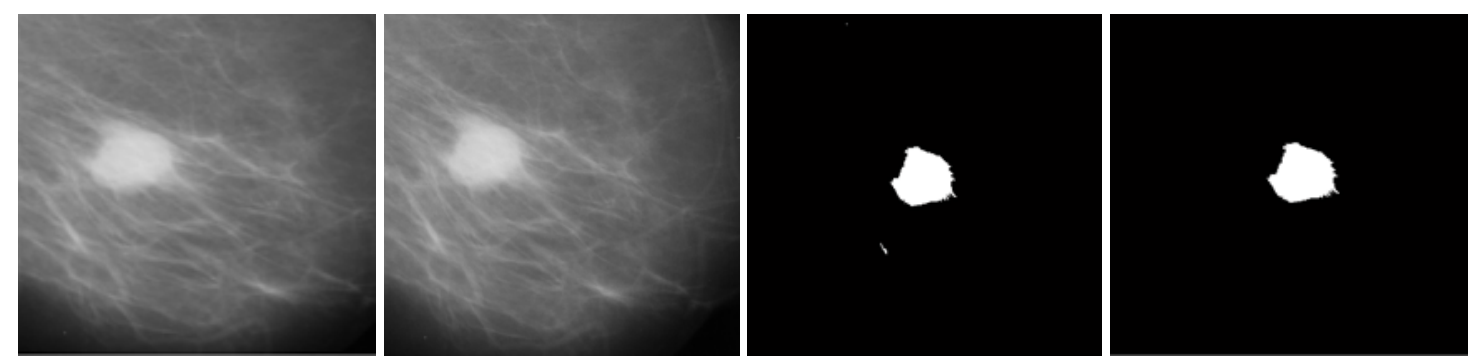

(a) Original image

(b) Preprocessed image

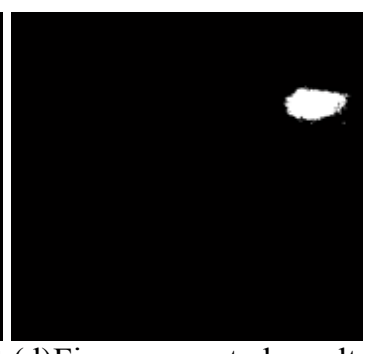

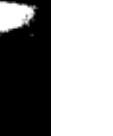



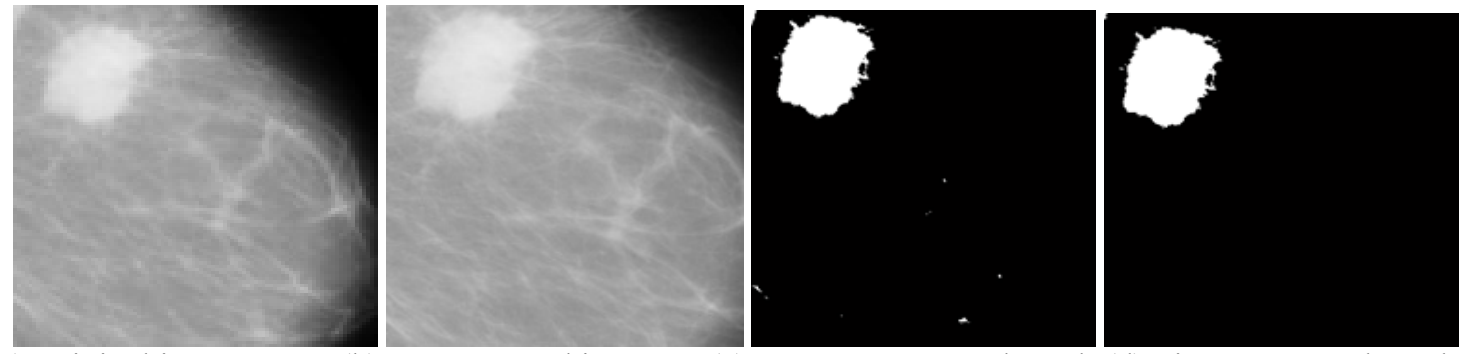

(a) Original image

(b) Preprocessed image

(c) Coarse segmented result

(d) Fine segmented result Image mdb184

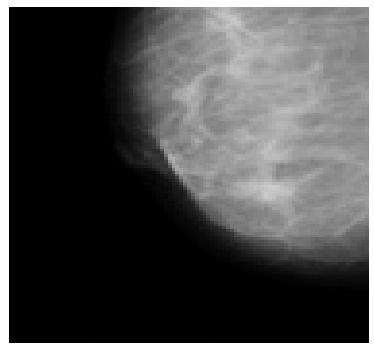

(a) Original image

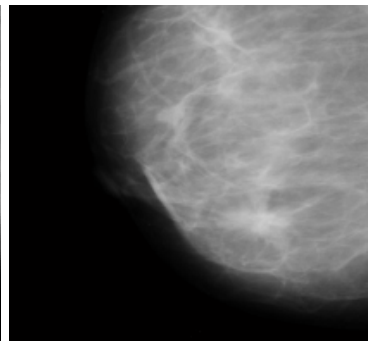

(b) Preprocessed image

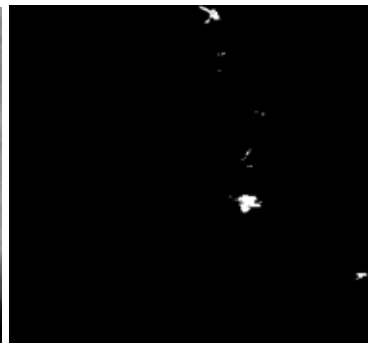

(c) Coarse segmented result mdb181

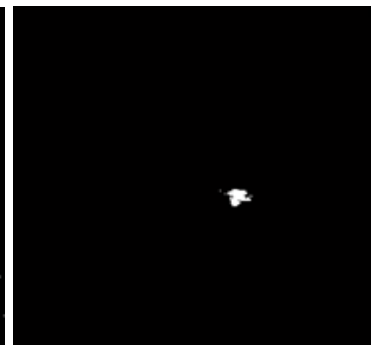

(d) Fine segmented result

\section{Image mdb181}

Figure 9. Example of segmentation results by the wavelet based adaptive windowing method of thresholding

Fig.8 (a) shows the original mammogram images. Then Fig.8 (b) shows the segmentation results for window based adaptive thresholding in which some normal tissues also segmented along with the tumours. Fig.9 (a) shows the original mammogram images. Then Fig.9(b) shows the preprocessed output.

Fig.9(c) shows the coarse segmented result. Fig.9 (d) shows the fine segmented result. The effective result can be obtained with small window size as $15^{*} 15$ and large window size as $128 * 128$ which is shown in Fig.9 (d).Therefore, the proposed algorithm obtained a good detection result.

\section{CONCLUSION}

In this paper, wavelet based adaptive windowing method is presented for the segmentation of bright targets in an image. Coarse segmentation is proposed by using wavelet based histogram thresholding where, the thereshold value is chosen by performing 1-D wavelet based analysis of PDFs of wavelet transformed images at different channels. Final segmented result is obtained by choosing threshold by using windowing method. The simulation results show that the proposed method is effective to segment the tumors in mammograms and it can also be used in other segmentation applications. Simulation results show that the proposed algorithms yield significantly superior image quality when it is compared to the Global thresholding method and window based adaptive thresholding method.

\section{REFERENCES}

[1].T.C.Wang, N.B. Karayiannis, "Detection of microcalcifications in digital mammograms using wavelets, Medical Imaging," IEEE Transactions, $17,498-509,1998$.

[2].H. Kobatake, M. Murakami, H. Takeo, and S. Nawano, "Computerized detection of malignant tumors on digital mammograms," IEEE Trans.Med. Imag., vol. 18, no. 5, pp. 369-378, May 1999.

[3].R.Mata, E.Nava,F. Sendra, "Microcalcifications detection using multi resolution methods, pattern Recognition,"'2000,proceeings, $15^{\text {th }}$ International Conference.4,344-347,2000.

[4].X. P. Zhang, "Multiscale tumor detection and segmentation in mammograms," in Proc. IEEE Int. Symp. Biomed. Imag., pp. 213-216, Jul. 2002.

[5].F. Fauci, S. Bagnasco, R. Bellotti, D. Cascio, C. Cheran, F. De Carlo, G. De Nunzio, M. E. Fantacci, G. Forni, A. Lauria, E. Lopez Torres, R. Magro, G. L. Masala, P. Oliva, M. Quarta, G. Raso, A. Retico, and S. Tangaro, "Mammogram segmentation by contour searching and mass lesions classification with neural network," IEEE Trans. Nucl. Sci., vol. 53, no. 5, pp. 2827-2833, Oct. 2006.

[6].Grady, L., "Random Walks for Image Segmentation," IEEE Transactions on PAMI 28(11), 1-17, 2006.

[7].Ersoy, I., Bunyak, F., Palaniappan, K., Sun, M., Forgacs, G., "Cell Spreading Analysis with Directed Edge Profile-Guided Level Set Active Contours," In: Metaxas, D., Axel, L., Fichtinger, G., Székely, G. (eds.) MICCAI 2008, Part I. LNCS, vol. 5241, pp. 376-383. Springer, Heidelberg, 2008. 
[8].A.Mencattini, M. Salmeri, R. Lojacono, M. Frigerio, and F. Caselli,"Mammographic images enhancement and denoising for breast cancer detection using dyadic wavelet processing," IEEE Trans. Instrum. Meas., vol. 57, no. 7, pp. 14221430, Jul. 2008.

[9]. Giovanni Palma, Isabelle Bloch, and Serge Muller "Spiculated Lesions and Architectural Distortions Detection in Digital Breast Tomosynthesis Datasets," IWDM 2010, LNCS 6136, pp. 712-719, 2010.

[10] S. Liu, C. F. Babbs, and E. J. Delp, "Multiresolution detection of speculated lesions in digital mammograms," IEEE Trans. Image Process., vol. 10, no. 6, pp. 874-884, Jun. 2001.

[11] G. M. te Brake and N. Karssemeijer, "Segmentation of suspicious densities in digital mammograms," Med. Phys., vol. 28, no. 2, pp. 259-266, Feb. 2001.

[12]Shengzhou $\mathrm{Xu} \&$ Hong Liu \& Enmin Song "Marker-Controlled Watershed for Lesion Segmentation in Mammograms," J Digital Imaging 24:754-763, 2011.

[13]M. Grgic et al. (Eds.): Rec. Advan. in Mult. Sig. Process. And Communication., SCI 231, pp. 631657, 2009.

[14]Umesh Adiga et al" High Throughput Analysis of Multispectral Images of Breast Cancer Tissue," IEEE Trans on image processing, vol.15,No.8, August 2006.

[15] H. S.Wu and J. Barba, "An efficient semiautomatic algorithm for cell contour extraction," J. Microscopy, vol. 179, pp. 270-276, 1995.

[16].N. H. Eltonsy, G. D. Tourassi, and A. S. Elmaghraby, "A concentric morphology model for the detection of masses in mammography," IEEE Trans. Med. Imag., vol. 26, no. 6, pp. 880889, Jun. 2007.

[17]A. Nedzved, S. Ablameyko, and I. Pitas, "Morphological segmentation of histology cell images," presented at the Int. Conf. Pattern Recognition, Barcelona, Spain, 2000.

[18]S. Schupp, A. Elmoataz, P. Herlin, and D. Bloyet, "Mathematical morphologic segmentation dedicated to quantitative immunohistochemistry," Anal. Quant. Cytol. Histol., vol. 23, no. 4, pp. 25767, 2001.

[19]H. S. Wu, J. Barba, and J. Gil, “A parametric fitting algorithm for segmentation of cell images," IEEE Trans. Biomed. Eng., vol. 45, no. 3, pp. 400-407, Mar. 1998.

[20].Wei Ping Li Junli, Zhao Shanxu,Lu Dongming,Chen Gang, "A Method of Detection Micro-Calcifications in Mammograms Using Wavelets and Adaptive Thresholds," The second International Conference on Bioinformatics and Biomedical Engineering, ICBBE 2008, pp.2361 2364, 2008.

[21].Pradhan, S Swaroop Patra, D Nanda, P Kumar, "Adaptive Thresholding Based Image Segmentation with Uneven Lighting Condition," the Third International Conference on Industrial and Information Systems, ICIIS, Kharagpur, December 8-10, 2008.

[22] S. Mallat, "A theory for multiresolution signal decomposition: The wavelet representation," IEEE Trans. Pattern Anal. Mach. Intell., vol. 11, no. 7, pp. 674-693, Jul. 1989.

[23]Grossman, A. and Morlet, J., "Decomposition of Hardy functions into square integrable wavelets of constant shape," SIAM J. Math. Anal., Vol. 15, No. 4, pp. 723-736, 1984.

[24]Garbay, "Image structure representation and processing: A discussion of some segmentation methods in cytology," IEEE Trans. Pattern Anal. Mach. Intell., vol. PAMI-8, pp. 140-146, 1986.

[25] Mallat, S., "A theory for multiresolution signals decomposition: The wavelet representation," IEEE Trans. Pattern Anal. Mach. Intell., Vol. 11, No. 7, pp. 674-693, 1989.

[26]Daubechies, I., "Orthonormal bases of compactly supported wavelets," Commun. Pure Appl. Math., Vol. 41, No. 7, pp. 909-996, 1988.

[27]Unser, M., Aldroubi, A., and Laine, A., "Special issue on wavelets in medical imaging," IEEE transactions on medical imaging, Vol. 22, No. 3, 2003.

[28]Weaver, J. B., Yansun, X., Healy, D. M., and Cromwell, L. D., "Filtering noise from images with wavelet transform," Magn. Reson. Med., Vol. 21, No. 2, pp. 288-295, 1991.

[29]X.P.Zhang and M. D. Desai, "Segmentation of bright targets using wavelets and adaptive thresholding," IEEE Trans. Image Process., vol. 10, no. 7, pp. 1020-1030, Jul. 2001.

[30]G.Kom, A.Tiedeu, and M. Kom, "Automated detection of masses in mammograms by local adaptive thresholding," Comput. Biol. Med.,vol. 37, no. 1, pp. 37-48, Jan. 2007.

[31]Maysam Shahedi B K1, Rassoul Amirfattahil, Farah Torkamani Azar and Saeed Sadri, "Accurate breast region detection in digital mammograms using a local adaptive thresholding method," Eight International Workshop on Image Analysis for Multimedia Interactive Services (WIAMIS'07), 2007.

[32]G.Kom, A. Tiedeu, and M. Kom, "Automated detection of masses in mammograms by local adaptive thresholding," Comput. Biol. Med.,vol. 37, no. 1, pp. 37-48, Jan. 2007.

[33]Brzakovic,D., Luo, X.M., Brzakovic, P., “An approach to automated detection of tumors in mammograms," IEEE Transactions on Medical Imaging 9(3), 233-241,1990.

[34]Ju Cheng Yang, Jin Wook Shin, and Dong Sun Park., "Comparing Study for Detecting Microcalcifications in Digital Mammogram Using Wavelets," LNCS 3177, pp. 409-415, 2004.

[35]A. Sarti, C. Solorzano, S. Lockett, and R. Malladi, "A geometric model for 3-D confocal image 
analysis," IEEE Trans. Biomed. Eng., vol. 47, no. 12, pp. 1600-1609, Dec. 2000.

G.Bharatha Sreeja, completed B.E (Electronics and Communication Engineering) in 2010 from Cape Institute of Technology, Anna University, Chennai. She is currently pursuing Final year M.E Communication Systems in Cape Institute of Technology, Anna University, Chennai. She has presented 4 papers in National and International Conferences. Her areas of interest include Medical Image Processing and Soft computing.

Dr.P.Rathika completed her B.E. (Electrical and Electronics Engineering) in 2001 from Dr.Sivanthi Aditanar College of Engineering, MS University and M.E. (Applied Electronics) in 2003 from Coimbatore Institute of Technology, Bharathiyar University. She did her Ph.D. (Electrical Engineering) in 2011 from Anna University, Chennai. She has got more than 8 years of teaching experience and 6 years of research experience. Her areas of interest include Soft computing, Power Quality, Digital Signal Processing, Solar PV system. She has published 4 papers international journals and 18 papers in conference proceedings.

Dr.D.Devaraj, completed his B.E (Electrical and Electronics Engineering) in 1992 and M.E (Power Systems) at Thiagarajar College of Engineering, Madurai, in 1992. He did his Ph.D (Power Systems) from IIT Madras, Chennai, in 2001. He has got more than 15 years of teaching experience and 15 years of research experience. He is a Ph.D Evaluator for Madurai Kamarajar University. He has guided 9 candidates for $\mathrm{Ph} . \mathrm{D}$ scholars and presently guiding more than 10 candidates for Ph.D scholars. His areas of interest include Power System Security, Neural Network, Genetic algorithm, Power System Optimization. He wrote 2 books on Power system Analysis for III year Electrical Engineering Students, Basic Electrical Engineering for I year Mechanical \& Bio Technology Students. He has published more than 40 papers in national journals and international journals and more than 100 papers in conference proceedings. 